\title{
Statyba
}

\section{KUHN-TUCKER CONDITIONS IN SHAKEDOWN PROBLEMS}

\section{J. Atkočiūnas}

To cite this article: J. Atkočiūnas (1996) KUHN-TUCKER CONDITIONS IN SHAKEDOWN

PROBLEMS, Statyba, 2:5, 14-28, DOI: 10.1080/13921525.1996.10531545

To link to this article: https://doi.org/10.1080/13921525.1996.10531545

册 Published online: 26 Jul 2012.

Submit your article to this journal \lceil

Џ Article views: 51 


\title{
УСЛОВИЯ КУНА-ТАККЕРА В ЗАДАЧАХ ПРИСПІОСОБЛЯЕМОСТИ
}

\author{
Ю.Ю. Аткочюнас
}

\section{1. Постановка задачи}

Напряженно-деформированное состояние (НДС) диссипативной системы в общем случае зависит от истории нагружения $\mathbf{F}(t) \quad\left(\mathbf{F}_{\text {inf }} \leq \mathbf{F}(t) \leq \mathbf{F}_{\text {sup }}\right.$, здесь $\mathbf{F}_{\text {inf }}, \mathbf{F}_{\text {sup }}$ - заданные пределы изменения нагрузки). Для приспосабливающихся конструхпий ее НДС в разных аспектах рассмотрено в работах [1-9] и др. Только для частного случая - для момента, близкого к разрушенио идеаљно упруполастической приспосабливающейся конструкапи, распределение действительных остаточнбх усилий $\mathbf{s}_{r}^{*}$ единственно для хаждой из историй нагружения $\mathbf{F}_{\text {inf }} \leq \mathbf{F}(t) \leq \mathbf{F}_{\text {sup }}$ (в этом случае коэффициент запаса по приспособляемости близок к 1). Однако и здесь могут существовать области, где пластические деформации $\Theta_{p}$ равны нулю. Тогда $\mathbf{S}_{r}$ в статически неопределимых частях конструкции может быть неединственным: напряженное состояние обуславливается только уравнениями равновесия $[A] \mathbf{S}_{r}=\mathbf{0}$. Экстремальный энергетический принцип минимума дополнительной энергии позволяет в момент, предшествуюшшй циклически-пластическому разрушению, из всех статически допустимых остаточных усилий определить именно $s_{r}^{*}$ (поведение конструкиии относится $\mathbf{x}$ голономному). Тогда для анализа НдС приспосабливающейся хонструкии появяется возможность прямого применения построеникх на основе экстремальных энергетических принцитов двойственных задач математического программирования (статическая и кинематическая формулировхи [7]). Встречающаяся вырожденность экстремальной задачи в статической формулировке (основные неизвестные $-\mathbf{S}_{r}$ ) указнвает на невозможность однозначного определения остаточных перемещений $\mathbf{u}_{r}$ и деформаций $\Theta_{r}$ при решении задачи анализа в кинематической формулировке. При уже известном векторе $\mathbf{s}_{r}^{*}$ проблема определения пределов изменения остаточных перемещений хонструхции $\mathbf{u}_{r, \text { inf }} \leq \mathbf{u}_{r}(t) \leq \mathbf{u}_{r, s u p}$ представляется в качестве задачи математического программирования. Ограчичениями эгой задачи служат условия Куна-Таккера цля задачи анализа в статической формулировке и энергетическое условие - неравенство, ограничивающее сверху обпую диссипацию энергии процессов достижения состояния приспособляемости $D$. Упомянутое энерпетическое ограничение позволяет исключить комбинаторные условия о дополняющей нежесткости и тем самым упростить решение задачи оценки остаточных перемещений.

\section{2. Математические модели экстремальных задач анализа НЦІС}

Дискретная модель конструкции получается расчленением ее иа $\zeta$ конечных элементов с общим числом $s$ расчетных сечений (множество их индексов $I$ ). Степень свободы $m$, число составляющих каждопо из векторов усилий $\mathbf{S}=\mathbf{S}_{r}+\mathbf{S}_{e}$ и деформаций $\Theta=\Theta_{r}+\Theta_{e}$ ранияется $n$ (ицдекс $e$ относится 
к векторам, получениым из уиругого расчета). Степень статической неогределимости $k_{0}=n-m$. Однако детально конечноэлементныс матрицы и зависимости в настоящей статье пе рассматриваются.

В качестве энсргетических границ достижения состояния приспособляемости конструкции выступают минимальное значение дополиительной энергии $\min \mathscr{F}^{\prime}\left(\mathbf{S}_{r}\right)=\min 0.5 \cdot \mathbf{S}_{r}^{T}[D] \mathbf{S}_{r}=a^{*}$ для статически догустимых остаточных усилий $S_{r}$ и максимальнее значение обцей диссипации энергии $D_{\max }$ для кинематически догустимых перемещений и $\mathbf{u}_{r}[8,9] .3 д е с ь ~[D]-$ матрища податливости. Для голономпых процессов диссипация энергии рассчитывается согласно формуле $D=\lambda^{T} \mathbf{C}$, где $\mathbf{C}, \lambda$ - векторы констант и множителей пластичности для нелинейньх условий текучести

$$
\mathbf{f}\left(\mathbf{S}_{r}+\mathbf{S}_{e}\right) \leq \mathbf{C}
$$

Как известно, статически догустимьй вектор $S_{r}$ удовлетворяет уравнениям равновесия $[A] \mathbf{S}_{r}=\mathbf{0}$ и условиям текучести (1). Порядок алгебраического оператора уравнений равновесия $[A]$ есть $(m \times n)$. Экстремальные угтугие усилия $S_{e j, \max }, S_{e j, m i n}$ являются линейньми функциями от известных $\mathbf{F}_{\text {inf }}, \mathbf{F}_{\text {sup }}$. Векторн $\mathbf{S}_{e j, \text { max }}, \mathbf{S}_{e j, \text { min }}$ определяют все вершины годографа упругих уситй $\mathbf{S}_{e}(t)$ (чере $j$ индехсируются их симметричные пары, их множество $J$ ). Так, в условиях (1) одновременно учіттываются все вершины годографа

$$
\begin{gathered}
\mathbf{f}\left(\mathbf{S}_{r}+\mathbf{S}_{e j, \text { max }}\right) \leq \mathbf{C}, \quad \mathbf{f}\left(\mathbf{S}_{r}+\mathbf{S}_{e j, \text { min }}\right) \leq \mathbf{C} \\
\text { यIR BCex } j \in J .
\end{gathered}
$$

Максимаљьое число вериин годографа $\bar{p}=2^{m}$, множество их индексов в даљнейшем будет обозначаться через $P$.

Принпип минимума дополнительной энергии приводит к статической формулировке задачи приспособляемости:

найти

$$
\min 0.5 \cdot \mathbf{S}_{r}^{T}[D] \mathbf{S}_{r}=a^{*}
$$

при условиях

$$
\begin{gathered}
{[A] \mathbf{S}_{r}=\mathbf{0}, \quad \mathbf{f}\left(\mathbf{S}_{r}+\mathbf{S}_{e j, \text { max }}\right) \leq \mathbf{C}, \quad \mathbf{f}\left(\mathbf{S}_{r}+\mathbf{S}_{e j, \text { min }}\right) \leq \mathbf{C}} \\
\text { для всех } j \in J .
\end{gathered}
$$

Решением задачи (2)-(3) отределяются вектор $\mathbf{S}_{r}^{*}$ и значение $\boldsymbol{a}^{*}$ (рис. 1). Вследствие положительной отределенности матрицы $[D]$ и вынуклости условий текучести (3) задача (2)-(3) имеет только единственное решение $\mathbf{s}_{r}$. Общее число активных, т. е. удовлетворяюшихся в качестве равенств условий текучести, не теряя обшности, обозначается через $k$ (максимальное их число равняется $s$, $i \in I$; при конкретном методе дискретизации могут учитываться условия на линиях стыка элементов и т.д.). В дальнейшем множество индексов активньх ограничений обозначается через $K$. Условия Куна-Таккера для задачи минимизации (2)-(3) (найти $\min \mathscr{F}^{\prime}\left(\mathbf{S}_{r}\right)=\min 0.5 \cdot \mathbf{S}_{r}^{T}[D] \mathbf{S}_{r}$ при ограничениях (3)) Ігринимают вид [10]:

$$
\begin{gathered}
\nabla \cdot\left(\mathbf{S}_{r}^{*}\right)+\sum_{j}\left[\nabla \mathbf{f}\left(\mathbf{S}_{r}^{*}+\mathbf{S}_{e j, \max }\right)\right]^{T} \lambda_{j, \text { max }}+\sum_{j}\left[\nabla \mathbf{f}\left(\mathbf{S}_{r}^{*}+\mathbf{S}_{e j, \min }\right)\right]^{T} \lambda_{j, \min }-[A]^{T} \mathbf{u}_{r}=\mathbf{0} \\
\lambda_{j, \max }^{T}\left[\mathbf{C}-\mathbf{f}\left(\mathbf{S}_{r}^{*}+\mathbf{S}_{e j, \max }\right)\right]=0, \quad \lambda_{j, \min }^{T}\left[\mathbf{C}-\mathbf{f}\left(\mathbf{S}_{r}^{*}+\mathbf{S}_{e j, \max }\right)\right]=0 \\
\lambda_{j, \max } \geq \mathbf{0}, \quad \lambda_{j, \min } \geq \mathbf{0}, \quad j \in J
\end{gathered}
$$


Здесь $[\nabla \mathbf{f}]$ - матрица градиентов вектор-функщий текучести (3), ее порядок (s $\times n)$; $\lambda_{j, \max }, \lambda_{j, \min }, \mathbf{u}_{r}$ - векторы множителей, физический смысл которьх может быть определен, исходя из кинематической формулировки задачи анализа. С учетом $\left(\mathbf{S}_{r}\right)=0.5 \cdot \mathbf{S}_{r}^{T}[D] \mathbf{S}_{r}$ условия Куна-Таккера принимают вид:

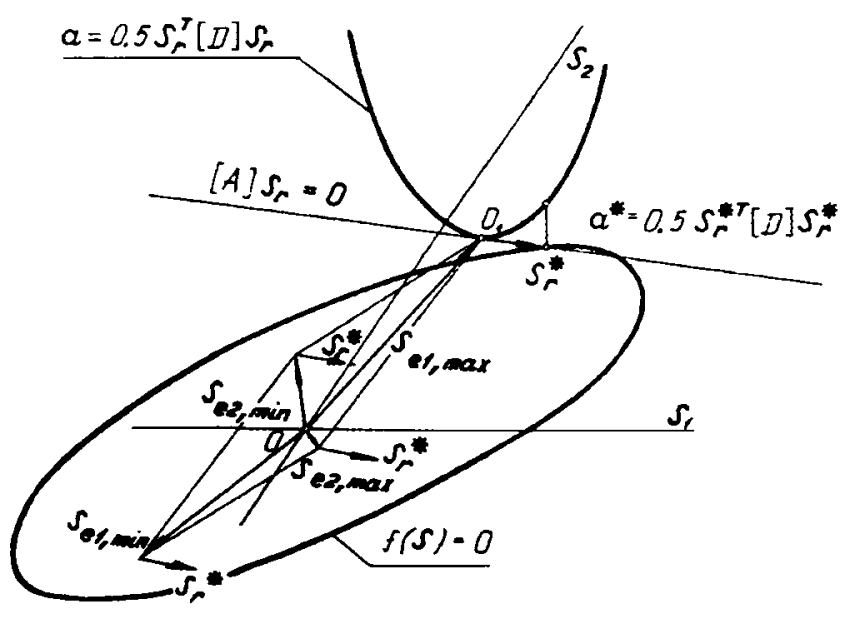

Рис. 1. К статической формулировке задачи анализа

$$
\begin{gathered}
{[D] \mathbf{S}_{r}^{*}+\sum_{j}\left[\nabla \mathbf{f}\left(\mathbf{S}_{r}^{*}+\mathbf{S}_{e j, \max }\right)\right]^{T} \lambda_{j, \max }+\sum_{j}\left[\nabla \mathbf{f}\left(\mathbf{S}_{r}^{*}+\mathbf{S}_{e j, \min }\right)\right]^{T} \lambda_{j, \min }-[A]^{T} \mathbf{u}_{r}=\mathbf{0}} \\
\lambda_{j, \max }^{T}\left[\mathbf{C}-\mathbf{f}\left(\mathbf{S}_{r}^{*}+\mathbf{S}_{e j, \max }\right)\right]=0, \quad \lambda_{j, \min }^{T}\left[\mathbf{C}-\mathbf{f}\left(\mathbf{S}_{r}^{*}+\mathbf{S}_{e j, \max }\right)\right]=0 \\
\lambda_{j, \max } \geq \mathbf{0}, \quad \lambda_{j, \min } \geq \mathbf{0} \\
\text { для всех } j \in J .
\end{gathered}
$$

Приппипу минимума полной потенциальой энергии отвечает кинематическая формулировка задачи анализа:

найти

$$
\begin{gathered}
\min \left\{0.5 \cdot \mathbf{S}_{r}^{T}[D] \mathbf{S}_{r}+\sum_{j} \lambda_{j, \max }^{T}\left[\nabla \mathbf{f}\left(\mathbf{S}_{r}+\mathbf{S}_{e j, \max }\right)\right] \mathbf{S}_{r}+\sum_{j} \lambda_{j, \min }^{T}\left[\nabla \mathbf{f}\left(\mathbf{S}_{r}+\mathbf{S}_{e j, \min }\right)\right] \mathbf{S}_{r}+\right. \\
\left.\quad+\sum_{j} \lambda_{j, \max }^{T}\left[\mathbf{C}-\mathbf{f}\left(\mathbf{S}_{r}+\mathbf{S}_{e j, \max }\right)\right]+\sum_{j} \lambda_{j, \min }^{T}\left[\mathbf{C}-\mathbf{f}\left(\mathbf{S}_{r}+\mathbf{S}_{e j, \min }\right)\right]\right\}=-a^{*}
\end{gathered}
$$

при условиях

$$
\begin{gathered}
{[D] \mathbf{S}_{r}+\sum_{j}\left[\nabla \mathbf{f}\left(\mathbf{S}_{r}+\mathbf{S}_{e j, \max }\right)\right]^{T} \lambda_{j, \max }+\sum_{j}\left[\nabla \mathbf{f}\left(\mathbf{S}_{r}+\mathbf{S}_{e j, \min }\right)\right]^{T} \lambda_{j, \min }-[A]^{T} \mathbf{u}_{r}=\mathbf{0}} \\
\boldsymbol{\lambda}_{j, \text { max }} \geq \mathbf{0}, \quad \lambda_{j, \min } \geq \mathbf{0} \\
\text { для всех } j \in J .
\end{gathered}
$$

Условия (7) ознатают геометрические уравнения $[A]^{T} \mathbf{u}_{r}=\Theta_{r}$. Решением задачи (6)-(7) определяются $\mathbf{s}_{r}^{*}, \mathbf{u}_{r}^{*}, \lambda_{j, \max }^{*}, \lambda_{j, \min }^{*}$. Дия оптимального плана задачи (6)-(7) согласно второй теореме двойственности теории математического Ігрограммирования 


$$
\lambda_{j, \max }^{* T}\left[\mathbf{C}-\mathbf{f}\left(\mathbf{S}_{r}^{*}+\mathbf{S}_{e j, \max }\right)\right]=0, \quad \lambda_{j, \min }^{* T}\left[\mathbf{C}-\mathbf{f}\left(\mathbf{S}_{r}^{*}+\mathbf{S}_{e j, \max }\right)\right]=0, \quad j \in J
$$

Векторы $\mathbf{u}_{r}^{*}, \lambda_{j, \max }^{*}, \lambda_{j, \min }^{*}$ удовлетворяют условиям Куна-Таккера (4) (или (5)). Для достижения состояния приспособляемости диссипация энергия $D=\lambda^{* T} \mathrm{C}$, где $\lambda^{*}=\sum_{j}\left(\lambda_{j, \max }^{*}+\lambda_{j, \min }^{*}\right)$. Заменой знака функции цели (6) на противоположный получается двойственная пара задач математического нелинейного программирования (2)-(3), (6)-(7):

найти

$$
\begin{aligned}
& \max "\left(\mathbf{S}_{r}, \mathbf{u}_{r}, \lambda\right)=\max \left\{-0.5 \cdot \mathbf{S}_{r}^{T}[D] \mathbf{S}_{r}-\sum_{j} \lambda_{j, \max }^{T}\left[\nabla \mathbf{f}\left(\mathbf{S}_{r}+\mathbf{S}_{e j, \max }\right)\right] \mathbf{S}_{r}-\sum_{j} \lambda_{j, \min }^{T}\left[\nabla \mathbf{f}\left(\mathbf{S}_{r}+\mathbf{S}_{e j, \min }\right)\right] \mathbf{S}_{r}-\right. \\
& \left.-\sum_{j} \lambda_{j, \max }^{T}\left[\mathbf{C}-\mathbf{f}\left(\mathbf{S}_{r}+\mathbf{S}_{e j, \max }\right)\right]-\sum_{j} \lambda_{j, \min }^{T}\left[\mathbf{C}-\mathbf{f}\left(\mathbf{S}_{r}+\mathbf{S}_{e j, \min }\right)\right]\right\}=a^{*}
\end{aligned}
$$

при условиях (7).

Для известных $\lambda_{j, \max }^{*}, \lambda_{j, \min }^{*}$ задача $(6)-(7)$ сводится $\mathbf{x}$ виду, отвечающему известному минимальному принципу Койтера [11]:

найти

$$
\min \left\{0.5 \cdot \mathbf{S}_{r}^{T}[D] \mathbf{S}_{r}+\sum_{j} \lambda_{j, \max }^{* T}\left[\nabla \mathbf{f}\left(\mathbf{S}_{r}+\mathbf{S}_{e j, \max }\right)\right] \mathbf{S}_{r}+\sum_{j} \lambda_{j, \min }^{* T}\left[\nabla \mathbf{f}\left(\mathbf{S}_{r}+\mathbf{S}_{e j, \min }\right)\right] \mathbf{S}_{r}\right\}=-a^{*}
$$

при условиях

$$
\begin{gathered}
{\left[D \mathbf{\mathbf { S } _ { r }}+\sum_{j}\left[\nabla \mathbf{f}\left(\mathbf{S}_{r}+\mathbf{S}_{e j, \max }\right)\right]^{T} \lambda_{j, \max }^{*}+\sum_{j}\left[\nabla \mathbf{f}\left(\mathbf{S}_{r}+\mathbf{S}_{e j, \min }\right)\right]^{T} \lambda_{j, \min }^{*}-[A]^{T} \mathbf{u}_{r}=\mathbf{0},\right.} \\
\lambda_{j, \max }^{* T}\left[\mathbf{C}-\mathbf{f}\left(\mathbf{S}_{r}+\mathbf{S}_{e j, \max }\right)\right]=0, \quad \lambda_{j, \min }^{* T}\left[\mathbf{C}-\mathbf{f}\left(\mathbf{S}_{r}+\mathbf{S}_{e j, \max }\right)\right]=0 \\
\lambda_{j, \max }^{*} \geq \mathbf{0}, \quad \lambda_{j, \min }^{*} \geq \mathbf{0} \\
\text { для всех } j \in J .
\end{gathered}
$$

В более компактной форме задача (8)-(9) обнчно представляется так: найти

$$
\min \left\{0.5 \cdot \mathbf{S}_{r}^{T}[D] \mathbf{S}_{r}+\mathbf{S}_{r}^{T} \Theta_{p}\right\}=-a^{*}
$$

при условиях

$$
[A]^{T} \mathbf{u}_{r}=[D] \mathbf{S}_{r}+\Theta_{p}
$$

где

$$
\begin{gathered}
\Theta_{p}=\sum_{j}\left[\nabla \mathbf{f}\left(\mathbf{S}_{r}+\mathbf{S}_{e j, \max }\right)\right]^{T} \lambda_{j, \text { max }}^{*}+\sum_{j}\left[\nabla \mathbf{f}\left(\mathbf{S}_{r}+\mathbf{S}_{e j, \min }\right)\right]^{T} \lambda_{j, \text { min }}^{*}, \\
\lambda_{j, \text { max }}^{* T}\left[\mathbf{C}-\mathbf{f}\left(\mathbf{S}_{r}+\mathbf{S}_{e j, \max }\right)\right]=0, \quad \lambda_{j, \min }^{* T}\left[\mathbf{C}-\mathbf{f}\left(\mathbf{S}_{r}+\mathbf{S}_{e j, \max }\right)\right]=0, \\
\lambda_{j, \text { max }}^{*} \geq \mathbf{0}, \quad \lambda_{j, \min }^{*} \geq \mathbf{0} \\
\text { для всех } j \in J .
\end{gathered}
$$


Для заданных пластических деформаций $\Theta_{p}^{*}$ второй член функции цели (10) задачи (10)-(12) является константой $C_{1}=\mathbf{S}_{r}^{T} \Theta_{p}^{*}$ и в конечном счете задача принимает вид: найти

$$
\min \left\{0.5 \cdot \mathbf{S}_{r}^{T}[D] \mathbf{S}_{r}+C_{1}\right\}=-a^{*}
$$

при условиях

$$
[A]^{T} \mathbf{u}_{r}=[D] \mathbf{S}_{r}+\Theta_{p}^{*}
$$

\section{3. Обобщенная задача Јагранжа}

Полную систему уравнений Ойлера-Лагранжа составляют ограничения математических моделей экстремальных задач в статической (2)-(3) и кинематической (4)-(5) формулировках с учетом условий о дополняющей нежесткости:

$$
\begin{gathered}
{[A] \mathbf{S}_{r}=\mathbf{0},} \\
{[D] \mathbf{S}_{r}+\Theta_{p}-[A]^{T} \mathbf{u}_{r}=\mathbf{0},} \\
-\mathbf{C}+\mathbf{f}\left(\mathbf{S}_{r}+\mathbf{S}_{e j, \max }\right) \leq \mathbf{0}, \quad-\mathbf{C}+\mathbf{f}\left(\mathbf{S}_{r}+\mathbf{S}_{e j, \min }\right) \leq \mathbf{0} ; \\
\lambda_{j, \text { max }}^{T}\left[\mathbf{C}-\mathbf{f}\left(\mathbf{S}_{r}+\mathbf{S}_{e j, \max }\right)\right]=0, \quad \lambda_{j, \text { min }}^{T}\left[\mathbf{C}-\mathbf{f}\left(\mathbf{S}_{r}+\mathbf{S}_{e j, \max }\right)\right]=0 \\
\lambda_{j, \text { max }} \geq \mathbf{0}, \quad \lambda_{j, \text { min }} \geq \mathbf{0} \\
\text { для Bсеx } j \in J .
\end{gathered}
$$

Здесь $\Theta_{p}-$ пластические деформации, входяшие в уравнения (7). Векторы $\mathbf{s}_{r}^{*}, \mathbf{u}_{r}^{*}, \lambda_{j, \max }^{*}, \lambda_{j, \min }^{*}$ являются решением системы зависимостей (15)-(19), которое полностью совпадает с решением двойственной пары задач математического программирования (2)-(3), (6)-(7).

Функиия

$$
\begin{gathered}
\mathscr{F}\left(\mathbf{S}_{r}, \mathbf{u}_{r}, \lambda\right)=0.5 \cdot \mathbf{S}_{r}^{T}[D] \mathbf{S}_{r}-\mathbf{u}_{r}^{T}[A] \mathbf{S}_{r}-\sum_{j} \lambda_{j, \max }^{T}\left[\mathbf{C}-\mathbf{f}\left(\mathbf{S}_{r}+\mathbf{S}_{e j, \max }\right)\right]- \\
\quad-\sum_{j} \lambda_{j, \min }^{T}\left[\mathbf{C}-\mathbf{f}\left(\mathbf{S}_{r}+\mathbf{S}_{e j, \min }\right)\right], \quad \lambda_{j, \max } \geq \mathbf{0}, \quad \lambda_{j, \min } \geq \mathbf{0}, \quad j \in J
\end{gathered}
$$

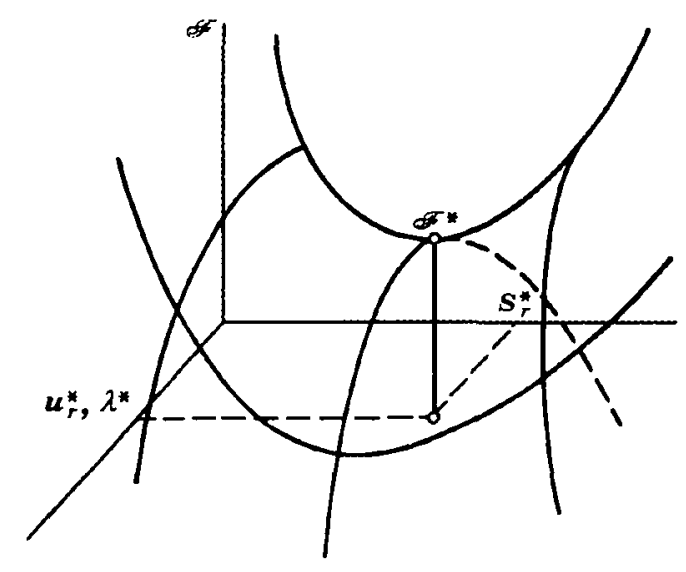

Рис. 2. Седловая функщия 
является потенциалом для системы зависимостей (15)-(19) [12]. Стационарная точка $\mathbf{s}_{r}^{*}$, $\mathbf{u}_{r}^{*}, \lambda_{j, \max }^{*}, \lambda_{j, \min }^{*}\left(\lambda^{*}=\sum_{j}\left(\lambda_{j, \text { max }}^{*}+\lambda_{j, \text { min }}^{*}\right)\right)$ функщии $\mathscr{F}_{r}\left(\mathbf{S}_{r}, \mathbf{u}_{r}, \lambda\right)$ есть решение системы (15)-(19): $F^{*}=\left(\mathbf{s}_{r}^{*}, \mathbf{u}_{r}^{*}, \lambda^{*}\right)$. Седловая фунхпия (20) является вьпухлой относительно $\mathbf{s}_{r}$ и вогнутой относительно $\mathbf{u}_{r}, \lambda$ (рис. $2[10,12]$ ). С использованием трансформаций Лежандра $[10,12]$ седловая функаия $\left(\mathbf{S}_{r}, \mathbf{u}_{r}, \lambda\right)$ (20) трансформируется либо только в выпуклую '(S $\left.\mathbf{S}_{r}\right)$, либо только в вогнугую " функии:

$$
\begin{aligned}
& \mathscr{F}^{\prime}=\mathscr{F}_{-} \mathbf{u}_{r}^{T} \delta \mathscr{F}_{\mathbf{u}_{r}}-\lambda^{T} \delta \mathscr{F}_{, \lambda}, \\
& \mathscr{F}^{\prime \prime}=\mathrm{S}_{r}^{T} \delta \mathscr{F}_{\mathrm{s}_{r} .} .
\end{aligned}
$$

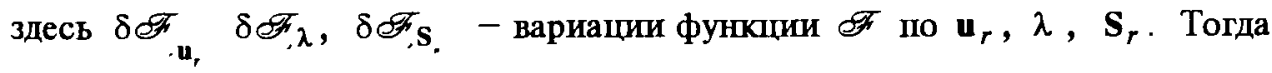

$\min _{\mathbf{S}_{r}} \max _{\mathbf{u}_{r}, \lambda \geq 0}=\min _{\mathbf{S}_{r}}=\max _{\mathbf{u}_{r}, \lambda \geq 0}$

и двойственная пара задач математического программирования (2)-(3), (6)-(7) соответственно Статическая формулировка

найти $\min \mathscr{7}^{\prime}$ при условиях $\delta \mathscr{F}_{\mathrm{u},}=\mathbf{0}, \delta \mathscr{F}_{\lambda} \leq \mathbf{0}$.

Кинематическая формулировка

найти $\max "$ при условиях $\delta$ f $_{s,}=\mathbf{0}, \lambda \geq 0$.

Здесь для простоты записи привлехался только вектор $\lambda \geq \mathbf{0}$, а не векторн $\lambda_{j, \max } \geq 0, \lambda_{j, \min } \geq 0$.

\section{O вьрохденной задаче анализа}

Для задачи (6)-(7) (или соответственно для задачи (15)-(19)), когда $k>k_{0}$, характерна возможность существования не одного вектора $\lambda^{*} \geq 0$, приводящего $\mathrm{x}$ тому же значению $a^{*}$. Следует подчерхнуть, что применяемый для решения нелинейной задачи (6)-(7) алторитм должен способствовать выделению $\lambda^{*}$, приводящему $\mathbf{k}$ максиматьному значению диссипации энергии $D_{\max }=\lambda^{* T} \mathrm{C}[10,13]$. Минимальное значение диссипации энергии $D_{\min }$ для достижения состояния приспособляемости (в том числе и в случае вырожденности задачи анализа) может быть определено решением задачи: найти $\min \lambda^{T} \mathrm{C}$ при условиях (15)-(19). Значительным упрощением решения этой задачи был бы предварительный учет в условиях (15)-(19) известного по решению задачи (2)-(3) вектора $\mathbf{S}_{r}^{*}$. Однако задача прямого определения $D_{\text {max }}$ : найти $\max \lambda^{T} \mathbf{C}$ при условиях (15)-(19), в общем случае теряет смысл из-за неограниченности функции цели такой задачи.

При наличия вырожденности задачи анализа в статической формулировке из кинематической формулировки (6)-(7) однозначно определить векторы $\Theta^{*}$, ч $^{*}$ не представляется возможнмм. Для выявления пределов изменения $\mathbf{u}_{r, \text { inf }}, \mathbf{u}_{r, \text { sup }}$ в дальнейшем и рассматривается соответствующая задача математического программирования (задача оценки остаточных перемещений). Как отмечалось вьше, решением задачи (2)-(3) (или задачи (6)-(7)) определяпося активные условия текучести $k$ и соответствуюшие им угтугие усипия $\mathrm{s}_{e}^{*}$ (в общем случае здесь могут учитываться интегральные условия текучести и т. Д.). Для всех $\mathbf{s}$ сечений (даже тех, в которых отсутствуют активные условия текучести) определению вектора $s_{e}^{*}$ способствует правило:

$$
\max _{p} f_{i}\left(\mathbf{S}_{n}^{*}+\mathbf{S}_{e i, p}\right)=f_{i}\left(\mathbf{S}_{n}^{*}+\mathbf{S}_{e i}^{*}\right), i \in I, p \in P .
$$


С привлечением векторов $\mathbf{S}_{r}^{*}$ и $\mathbf{S}_{e}^{*}$ определяется матрица градиентов $\left[\nabla \mathbf{f}\left(\mathbf{S}_{r}^{*}+\mathbf{S}_{e}^{*}\right)\right]=\left[\nabla \mathbf{f}^{*}\right]$. Тогда пластические деформации $\Theta_{p}$ определяются значительно проще, так как из рассмотрения предварительно искпючаются нулевые составляюшие векторов $\lambda_{j, \max }^{*}, \lambda_{j, \min }^{*}$ :

$$
\Theta_{p}=\left[\nabla \mathbf{f}\left(\mathbf{S}_{r}^{*}+\mathbf{S}_{e}^{*}\right)\right]^{T} \lambda, \quad \lambda \geq \mathbf{0}, \quad \lambda^{T}\left[\mathbf{C}-\mathbf{f}\left(\mathbf{S}_{r}^{*}+\mathbf{S}_{e}^{*}\right)\right]=0 .
$$

Среди условий текучести $\mathrm{f}\left(\mathrm{S}_{r}^{*}+\mathrm{S}_{e}^{*}\right) \leq \mathrm{C} k$ из них удовлетворяются в хачестве строгих равенств (множество их индехсов $K$ ). Поэтому хомбинаторнње условия о дополняющей нежесткости

$$
\lambda^{T}\left[\mathbf{C}-\mathbf{r}\left(\mathbf{S}_{r}^{*}+\mathbf{S}_{e}^{*}\right)\right]=0
$$

для них можно было бы предварительно исклочить.

\section{5. Уравнения совместности остаточных деформаций}

Продолжается исследование условий Куна-Таккера (4) для задачи анализа в статической формултровке (2)-(3):

$$
\begin{gathered}
{[D] \mathbf{S}_{r}+\sum_{j}\left[\nabla \mathbf{f}\left(\mathbf{S}_{r}+\mathbf{S}_{e j, \max }\right)\right]^{T} \lambda_{j, \max }+\sum_{j}\left[\nabla \mathbf{f}\left(\mathbf{S}_{r}+\mathbf{S}_{e j, \min }\right)\right]^{T} \lambda_{j, \min }-[A]^{T} \mathbf{u}_{r}=\mathbf{0},} \\
\lambda_{j, \max }^{T}\left[\mathbf{C}-\mathbf{f}\left(\mathbf{S}_{r}+\mathbf{S}_{e j, \max }\right)\right]=0, \quad \lambda_{j, \min }^{T}\left[\mathbf{C}-\mathbf{f}\left(\mathbf{S}_{r}+\mathbf{S}_{e j, \max }\right)\right]=\mathbf{0}, \\
\lambda_{j, \max } \geq \mathbf{0}, \quad \lambda_{j, \min } \geq \mathbf{0} \\
\text { для Bсеx } j \in J .
\end{gathered}
$$

Уравнения совместности остаточных деформаций могут быть получены из условий Куна-Такхера искпочением из них остаточнкх перемещений $\mathbf{u}_{r}[14]$ :

$$
[B] \Theta_{p}=\left[B_{r}\right] \mathbf{S}_{r},
$$

где

$$
\begin{gathered}
\Theta_{p}=\sum_{j}\left[\nabla \mathbf{f}\left(\mathbf{S}_{r}+\mathbf{S}_{e j, \max }\right)\right]^{T} \lambda_{j, \max }+\sum_{j}\left[\nabla \mathbf{f}\left(\mathbf{S}_{r}+\mathbf{S}_{e j, \min }\right)\right]^{T} \lambda_{j, \text { min }}, \\
\lambda_{j, \max }^{T}\left[\mathbf{C}-\mathbf{f}\left(\mathbf{S}_{r}+\mathbf{S}_{e j, \max }\right)\right]=0, \quad \lambda_{j, \min }^{T}\left[\mathbf{C}-\mathbf{f}\left(\mathbf{S}_{r}+\mathbf{S}_{e j, \max }\right)\right]=0, \\
\lambda_{j, \text { max }} \geq \mathbf{0}, \quad \lambda_{j, \min } \geq \mathbf{0} \\
\text { для всех } j \in J .
\end{gathered}
$$

Порядок матрищ $[B],\left[B_{r}\right]$ таков $\left(k_{0} \times n\right)$ :

$$
[B]=\left[\left[A^{\prime \prime}\right]^{T}\left(\left[A^{\prime}\right]^{T}\right)^{-1},-[\mathrm{I}]\right], \quad\left[B_{r}\right]=-\left[A^{\prime \prime}\right]^{T}\left(\left[A^{\prime}\right]^{T}\right)^{-1}\left[D^{\prime}\right]+\left[D^{\prime \prime}\right]
$$

Считается, что квадратная порядка $(m \times m)$ матрица $\left[A^{\prime}\right]^{T}$ выбрана таким образом, что суцествует ее обратная (матрица $\left[D^{\prime}\right]$ отвечает матрице $\left[A^{\prime}\right]^{T}$ ).

Приппи минимума дополнительной энерги (или двойственный ему приппип минимума полной потенциальной энергии) и уравнения совместности остаточнњх деформаций тождественны между собой : 


$$
\begin{gathered}
{[B] \Theta_{p}=\left[B_{r}\right] \mathbf{S}_{r}^{*},} \\
\Theta_{p}=\left[\nabla \mathbf{f}\left(\mathbf{S}_{r}^{*}+\mathbf{S}_{e}^{*}\right)\right]^{T} \lambda, \quad \lambda \geq \mathbf{0}, \quad \lambda^{T}\left[\mathbf{C}-\mathbf{f}\left(\mathbf{S}_{r}^{*}+\mathbf{S}_{e}^{*}\right)\right]=0 .
\end{gathered}
$$

Анализ полученных уравнений (26) продолжается с принятия в них $\lambda$ :

$$
\begin{gathered}
{\left[B_{\lambda}^{*}\right] \lambda=\left[B_{r}\right] \mathbf{S}_{r}^{*}, \quad \lambda \geq \mathbf{0},} \\
\lambda^{T}\left[\mathbf{C}-\mathbf{f}\left(\mathbf{S}_{r}^{*}+\mathbf{S}_{e}^{*}\right)\right]=0 .
\end{gathered}
$$

Здесь $\left[B_{\lambda}^{*}\right]=[B]\left[\nabla \mathbf{f}^{*}\right]^{T}$, ее порядок $\left(k_{0} \times s\right)$. Неизвестными в системе зависимостей (27)-(28) являотся векторы $\lambda \geq 0$. В результате анализа (27)-(28) становится очевидным цель определения вектора $S_{\&}^{*}$, а именно: при формировании уравнений совместности заранее из рассмотрения исключается значительная часть условий текучести, не относяпихся к числу активных. Вследствие этого порядох матричы $\left[B_{\lambda}^{*}\right]$ есть $\left(k_{0} \times s\right)$, а не $\left(k_{0} \times \bar{p} \times s\right)$. Если число активньх условий текучести $k=s(K=I)$, то из системы (27)-(28) можно исключить условия (28). В итоге получается

$$
\left[B_{\lambda}^{*}\right] \lambda=\left[B_{r}\right] \mathbf{S}_{r}^{*}, \quad \lambda \geq \mathbf{0}
$$

Вирожденность задачи (2)-(3), қак отмечено выше, может иметь место, если $k>k_{0}$, т. е. если qисло активных условий текучести превышает степень статической неопределимости хонструкиии. В этом случае лобое решение $\lambda \geq 0$ системы (27)-(28) всегда удовлетворяет получаемому из зависимости

$$
\mathbf{S}_{r}^{* T}[A]^{T} \mathbf{u}_{r}=\mathbf{S}_{r}^{* T}[D] \mathbf{S}_{r}^{*}+\mathbf{S}_{r}^{* T} \boldsymbol{\theta}_{p}
$$

энергетическому равенству $-S_{r}^{* T} \theta_{p}=2 a^{*}$. В теории математическото программирования энергетическое равенство $-\mathrm{S}_{r}^{* T} \theta_{p}=2 a^{*}$ носит название "первой теоремы двойственности". Следовательно, энергетическая граница приспособляемости $a^{*}$ для определения векторов $\mathbf{u}_{r, \text { inf }}, \mathbf{u}_{r, \text { sup }}$ с привлечением только геометрических уравнений (7) самостоятельного значения не имеет. Только каждый из $\bar{v}$ базнсных векторов $\lambda_{v}^{*} \geq 0$ системы (27)-(28) отвечает состоянию приспособляемости конструкиии и энергетическому ограничению $\lambda_{v}^{-\tau} \mathrm{C} \leq D_{\max }, \quad v=1,2, \ldots, \vec{v}$. Очевидно, что число ненулевых составиюших для каждого базисного вектора $\lambda_{v}^{*}$ не превышает степени сгатической неопределимости конструкции $k_{0}$. Только в этом случае диссипация энергии для достижения состояния приспособляемости $D$ ограничена сверху. После разработки системы уравнений (27)-(28) рассмотренная в подразделе 4 задача определения $D_{\min }$ может бить представлена так:

найти

при условиях

$$
\min \lambda^{T} \mathbf{C}
$$

$$
\left[B_{\lambda}^{*}\right] \lambda=\left[B_{r}\right] \mathbf{S}_{r}^{*}, \quad \lambda \geq \mathbf{0}, \quad \lambda^{T}\left[\mathbf{C}-\mathbf{f}\left(\mathbf{S}_{r}^{*}+\mathbf{S}_{e}^{*}\right)\right]=0
$$


Это учитывалось в нашпх исследованиях [14] при построении математических моделей задач оптимизации в условиях приспособляемости с учетом как прочностных, так и жесткостньх огранпченпй.

И еще о системе зависимостей (26). Математическая модель задачи анализа в статической формулировке (2)-(3) значительно упроцается, если предварительно из условий-ограничений искпючаются уравнения равновесия $[A] \mathbf{S}_{r}=\mathbf{0}\left(\left[A^{\prime}\right] \mathbf{S}_{r}^{\prime}+\left[A^{\prime \prime}\right] \mathbf{S}_{r}^{\prime \prime}=\mathbf{0}\right)$ :

найти

$$
\min 0.5 \cdot \mathbf{S}_{r}^{\prime \prime T}[\widetilde{D}] \mathbf{S}_{r}^{\prime \prime}=a^{*}
$$

при условиях

$$
\begin{gathered}
\mathbf{f}\left(-[B] \mathbf{S}_{r}^{\prime \prime}+\mathbf{S}_{e j, \max }\right) \leq \mathbf{C}, \quad \mathbf{f}\left(-[B] \mathbf{S}_{r}^{\prime \prime}+\mathbf{S}_{e j, \min }\right) \leq \mathbf{C} \\
\text { дIя Bcex } j \in J .
\end{gathered}
$$

Здесь $[\widetilde{D}]=[B][D][B]^{T}$, а вектор $\mathbf{S}_{r}=-[B]^{T} \mathbf{S}_{r}^{\prime \prime}$. Условия Куна-Таккера для задачи $(29)-(30)$ принимают вид:

$$
\begin{gathered}
{[\widetilde{D}] \mathbf{S}_{r}^{\prime \prime *}+\sum_{j}\left[\nabla \mathbf{f}^{\prime \prime}\left(-[B] \mathbf{S}_{r}^{\prime \prime *}+\mathbf{S}_{e j, \max }\right)\right]^{T} \lambda_{j, \max }+\sum_{j}\left[\nabla \mathbf{f}^{\prime \prime}\left(-[B] \mathbf{S}_{r}^{\prime \prime *}+\mathbf{S}_{e j, \min }\right)\right]^{T} \lambda_{j, \min }=\mathbf{0}} \\
\lambda_{j, \max }^{T}\left[\mathbf{C}-\mathbf{f}\left(-[B] \mathbf{S}_{r}^{\prime \prime}+\mathbf{S}_{e j, \max }\right)\right]=0, \quad \lambda_{j, \min }^{T}\left[\mathbf{C}-\mathbf{f}\left(-[B] \mathbf{S}_{r}^{\prime \prime *}+\mathbf{S}_{e j, \max }\right)\right]=0, \\
\lambda_{j, \max } \geq \mathbf{0}, \quad \lambda_{j, \min } \geq \mathbf{0} \\
\text { для всех } j \in J .
\end{gathered}
$$

Здесь $\left[\nabla f^{\prime \prime}\right]$ - матрища градиентов вектор-функпий текучести (30) при наличии неизвестнкх $\mathbf{S}_{r}^{\prime \prime}$; еe порядок $\left(s \times k_{0}\right)$. С учетом вектора $\mathbf{S}_{e}^{*}$ условия Куна-Такхера (31)-(32) представляотся так:

$$
\begin{gathered}
{[\widetilde{D}] \mathbf{S}_{r}^{\prime \prime *}+\left[\nabla \mathbf{f}^{\prime \prime}\left(-[B] \mathbf{S}_{r}^{\prime \prime *}+\mathbf{S}_{e}^{*}\right)\right]^{T} \lambda=\mathbf{0}} \\
\lambda^{T}\left[\mathbf{C}-\mathbf{f}\left(-[B] \mathbf{S}_{r}^{\prime *}+\mathbf{S}_{e}^{*}\right)\right]=0, \lambda \geq \mathbf{0}
\end{gathered}
$$

Уравнения (33)-(34) представляют собой зависимости (26), так как

$$
\begin{gathered}
-\left[\nabla \mathbf{f}^{\prime \prime}\left(-[B] \mathbf{S}_{r}^{\prime *}+\mathbf{S}_{e}^{*}\right)\right]^{T} \lambda=[B]\left[\nabla \mathbf{f}\left(\mathbf{S}_{r}^{*}+\mathbf{S}_{e}^{*}\right)\right]^{T} \lambda \\
{\left[B_{r}\right] \mathbf{S}_{r}^{*}=-\left[B_{r}\right][B]^{T} \mathbf{S}_{r}^{\prime *}=[\tilde{D}] \mathbf{S}_{r}^{\prime *}}
\end{gathered}
$$

и нетрудно заметить, что

$$
\left[B_{\lambda}^{*}\right]=-\left[\nabla \mathbf{f}^{\prime \prime}\left(-[B] \mathbf{S}_{r}^{\prime *}+\mathbf{S}_{e}^{*}\right)\right]^{T}
$$

В заключении подраздела матрица $[B]$ основных уравнений совместности деформации $\Theta$ строится с привлечением известных в линейной алгебре правосторонней псевдообратной матрипы $\left[A_{p}\right]^{-1}$ и матрищы ядра $\left[A_{p}^{0}\right]$. Статически возможный вехтор усилий $\mathbf{Q}$ для уравнений равновесия 
$\left[A_{q}\right] \mathbf{Q}=\mathbf{F}$ (норядок матрицы $\left.\left[A_{q}\right] \operatorname{ecrs}(m \times \widetilde{n}), \widetilde{n} \geq n\right)$ выражается так: $\mathbf{Q}=\left[A_{p}\right]^{-1} \mathbf{F}+\left[A_{p}^{0}\right] \mathbf{Q}^{\prime \prime}$.

Здесь

$$
[B]=\left[\left[A^{\prime \prime}\right]^{T}\left(\left[A^{\prime}\right]^{T}\right)^{-1},-[\mathrm{I}]\right],\left[A_{p}\right]^{-T}=\left[\left[A^{\prime \prime}\right]^{T}\left(\left[A^{\prime}\right]^{T}\right)^{-1},-[\mathrm{I}]\right] .
$$

Тогда последовательность оиераториых действий:

$$
\left[A_{q}\right]\left(\left[A_{p}\right]^{-1} \mathbf{F}+\left[A_{p}^{0}\right] \mathbf{Q}^{\prime \prime}\right)=\mathbf{F}, \quad\left[A_{q}\right]\left[A_{p}^{0}\right] \mathbf{Q}^{\prime \prime}=\mathbf{0}
$$

с учетом того, что $\left[A_{q}\right]\left[A_{p}^{0}\right]=[0]$ и $\left[A_{p}^{0}\right]^{T}\left[A_{q}\right]^{T} \mathbf{u}=\mathbf{0}$, приводит к основному уравнению совместности деформаций $\left[A_{p}^{0}\right]^{T} \Theta=0$. Нетрудно заметить, что $-\left[A_{p}^{0}\right]^{T}=[B]$. В рамках допуцения о малых перемещениях матрища $\left[A_{p}^{0}\right]$ не зависит от стадии работы конструкции. Следовательно, в качестве составляющих вектора $\Theta$ могут (в контексте согласования с вектором $\mathbf{Q}$ ) выступить упругие, остаточные, суммарные, в том числе и связанные с жестким перемещением, деформации конструкции.

\section{6. Математическпе модели задач оденки остаточных перемещений}

Вначале рассматриваются геометрические уравнения

$$
[A]^{T} \mathbf{u}_{r}^{*}=[D] \mathbf{S}_{r}^{*}+\Theta_{p}^{*},
$$

где

$$
\Theta_{p}^{*}=\left[\nabla \mathbf{r}\left(\mathbf{S}_{r}^{*}+\mathbf{S}_{e}^{*}\right)\right]^{T} \lambda^{*}, \quad \lambda^{*} \geq \mathbf{0}, \quad \lambda^{* T}\left[\mathbf{C}-\mathbf{f}\left(\mathbf{S}_{r}^{*}+\mathbf{S}_{e}^{*}\right)\right]=0
$$

конструкиии, приспособившейся $\mathbf{K}$ заданному повторно-переменному нагружению $\mathbf{F}_{\text {inf }} \leq \mathbf{F}(t) \leq \mathbf{F}_{\text {sup }}$. Определение остаточного перемещения $\dot{u}_{n}^{*}, i=1,2, \ldots, m$ приспособившейся конструхщии при единственном распределении $\mathbf{S}_{r}^{*}, \Theta_{p}^{*}$ основнвается на известной теореме Бетти: а именно, х заданной хонструхии по $i$-му направленипо прикладыпвается единичная сила $\bar{F}_{i}=1$, обуславливающая возникновение в ней упругих усилий $\mathbf{S}_{e}$. Умножением обеих сторон уравнений (36) на вехтор $\mathbf{S}_{e}$ получается

$$
\ddot{u_{n}^{*}}=\mathbf{S}_{e}^{T}[D] \mathbf{S}_{r}^{*}+\mathbf{S}_{e}^{T} \Theta_{p}^{*}=\mathbf{S}_{e}^{T} \Theta_{p}^{*},
$$

так как $\mathbf{S}_{e}^{T}[D] \mathbf{S}_{r}^{*}=0$. Поступая аналогичным образом, в конечном счете для всех $u_{n}^{\bullet}, i=1,2, \ldots, m$ получается зависимость:

$$
\mathbf{u}_{r}^{*}=[\alpha]^{T} \Theta_{p}^{*}=[\bar{H}] \Theta_{p}^{*} .
$$

Здесь $[\alpha]$ - матрица влияния усилий упругого расчета конструхциг; $[\bar{H}]=[\alpha]^{T}$ - матрица влияния остаточных перемешений. С другой стороны, зависимость (38) для известных деформаший $\Theta_{p}^{*}$ может быть получена (как и формула для определения $\left.S_{r}^{*}[14]\right)$ из системы уравнений (15)-(16):

$$
\mathbf{u}_{r}^{*}=\left([A][D]^{-1}[A]^{T}\right)^{-1}[A][D]^{-1} \Theta_{p}^{*}=[\alpha]^{T} \Theta_{p}^{*},
$$




$$
\mathbf{S}_{r}^{*}=\left\{[D]^{-1}[A]^{T}\left([A][D]^{-1}[A]^{T}\right)^{-1}[A][D]^{-1}-[D]^{-1}\right\} \Theta_{p}^{*}=\left\{[\alpha][A][D]^{-1}-[D]^{-1}\right\} \Theta_{p}^{*}=[\bar{G}] \Theta_{p}^{*}
$$

Возврашаясь $\mathbf{к}$ теореме Бетти, следует отметить, что единичная сила $\bar{F}_{i}=1$ может действовать хак на заданную конструкцию, тах и на основную систему метода сил или даже на систему с частично снихенной степенью статической неогределимости $k_{0}$ (статически квазиогределимая система). В последних двух случаях вектор статически возможншх упругих усилий от единичной силы обозначается через $\overline{\mathrm{S}}_{e}$ и

$$
u_{r i}^{*}=\overline{\mathbf{S}}_{e}^{T}[D] \mathbf{S}_{r}^{*}+\overline{\mathbf{S}}_{e}^{T} \Theta_{p}^{*}
$$

Здесь для систем, согласованншх с вектором $\Theta_{p}^{*}$, член $\overline{\mathbf{S}}_{e}^{T} \Theta_{p}^{*}$ может быть равен нулю. Для вектора $\mathbf{u}_{r}^{*}$ в целом формула (40) принимает вид

$$
\mathbf{u}_{r}^{*}=[\bar{\alpha}]^{T}[D] \mathrm{S}_{r}^{*}+[\bar{\alpha}]^{T} \Theta_{p}^{*} .
$$

Матрица $[\bar{\alpha}]^{T}$ отличается ог $[\alpha]^{T}$ наличием в ней нулевнх столбцов (для статически определимой системы $[\bar{\alpha}]=\left[A_{p}\right]^{-1}$ ). Из формулы (41) формально нетрудно получить зависимость (38). С этой целью правая сторона формулы (41), используя выражение $\mathbf{S}_{r}$ по зависимости (39), сводится $\mathbf{x}$ виду:

$$
[\bar{\alpha}]^{T}[D]\left\{[D]^{-1}[A]^{T}\left([A][D]^{-1}[A]^{T}\right)^{-1}[A][D]^{-1}-[D]^{-1}\right\} \Theta_{p}^{*}+[\bar{\alpha}]^{T} \Theta_{p}^{*}=[\alpha]^{T} \Theta_{p}^{*}
$$

Формула (41) может быть интерпретирована и в контексте теометрических уравнений (36):

$$
\mathbf{u}_{r}^{*}=\left(\left[A^{\prime}\right]^{T}\right)^{-1}\left[D^{\prime}\right] \mathbf{S}_{r}^{*}+\left(\left[A^{\prime}\right]^{T}\right)^{-1} \Theta_{p}^{* *}
$$

Формула (42) позволяет обосновать получение хомпьютерных ресурсов из-за снихения размеров соответствующих матриц (исключение нулевых столбцов в матрицах по системе (41)).

Делается обобщение. Уравнения метода сил $\left[\beta_{q}\right] \mathbf{S}^{\prime \prime}+\left[\beta_{p}\right] \mathbf{F}=\mathbf{0}$ позволяют определить $\mathbf{S}$ :

$$
\mathbf{S}=[\alpha] \mathbf{F}=\left(\left[A_{p}\right]^{-1}-\left[A_{p}^{0}\right]\left[\beta_{q}\right]^{-1}\left[\beta_{p}\right]\right) \mathbf{F}, \quad \text { где }\left[\beta_{q}\right]=\left[A_{p}^{0}\right]^{T}[D]\left[A_{p}^{0}\right], \quad\left[\beta_{p}\right]=\left[A_{p}^{0}\right]^{T}[D]\left[A_{p}\right]^{-1},
$$

а исходные уравнения равновесия $[A] \mathbf{S}=\mathbf{F}$. Используя полученное выражение матрицы влияния $[\alpha]$, вышеприведеншые выводы относительно перемещений могут быть получены, исходя из определений матрищ $\left[A_{p}\right]^{-1},\left[A_{p}^{0}\right]$.

Если для состояния приспособляемости вектор $\Theta_{p}^{*}-$ единственный (задача (2)-(3) невырождена), составляющие вектора $\mathbf{u}_{r}^{*}$ согласно формулам (36) или (42) определяются единственным образом и не зависят от подбора матришы $\left[A^{\prime}\right]^{T}$.

В случае вырожденности задачи анализа определяются только пределы изменения $\mathbf{u}_{r, \text { inf }}, \mathbf{u}_{r, \text { sup }}$ остаточных перемещений $\mathbf{u}_{r}=\left[H^{\bullet}\right] \lambda$ в момент, предшествуюший циклически-пластическому разрушению приспособившейся конструкции. Здесь $\left[H^{*}\right]=[\alpha]^{T}\left[\nabla \mathbf{f}^{*}\right]-$ матрица влияпия остаточных 
перемещений, приуроченная $\mathbf{k}$ вектору пластических множителей $\lambda \geq 0$. Базисный вектор пластических множителей $\lambda_{v}^{*} \geq 0$ системы (27)-(28) отвечает статически определимой (квазиопределимой) системе. Для отдельньх составляоцих векторов $\mathbf{u}_{r, \text { inf }}^{*}, \mathbf{u}_{r, \text { sup }}^{*}$ следует выделить наиболее "податливые" статически определимые (или квазиопределимые) системы. Поэтому для хахдого базисного вектора $\lambda_{v}^{*} \geq 0$ системы $(27)-(28)$ определяются векторы $\mathbf{u}_{r, v}^{*}=\left[H^{*}\right] \lambda_{v}^{*}, \quad v=1,2, \ldots, \bar{v}$. Простнм перебором составляюцих по всем векторам $\mathbf{u}_{r, v}^{*}$ определяются векторы пределов изменения остаточных перемещений $\mathbf{u}_{r, \text { inf }}^{*}, \mathbf{u}_{r}^{*}$, sup. Такой же результат получается решением следующей задачи математического программирования для хаждой $i$-й составляощей вектора перемещений $u_{n}$ :

найти

$$
\max _{\min } \mathbf{H}_{i}^{*} \lambda^{*}=\left[\begin{array}{c}
u^{*}{ }_{n, \text { sup }} \\
u_{i, \text { inf }}^{\bullet}
\end{array}\right], i=1,2, \ldots, m
$$

при условиях

$$
\left[B_{\lambda}^{*}\right] \lambda^{*}=\left[B_{r}\right] \mathbf{S}_{r}^{*}, \quad \lambda^{*} \geq \mathbf{0}, \quad \lambda^{* T}\left[\mathbf{C}-\mathbf{f}\left(\mathbf{S}_{r}^{*}+\mathbf{S}_{e}^{*}\right)\right]=0
$$

Неизвестними здесь являотся базнснше векторы $\lambda_{v}^{*} \geq 0$ (в противном случае $D \rightarrow \infty$ и функция цели (43) задачи (43)-(44) будет не ограничена). Приведенная методика корректного решения вкрохденной задачи анализа трудоемка в связи с многоразовьм определением базиснбх векторов $\lambda_{v}$.

Пределы $\quad \mathbf{u}_{r, \text { inf }}, \mathbf{u}_{r, \text { sup }} \quad$ тахске, что $\quad \mathbf{u}_{r, \text { inf }} \leq \mathbf{u}_{r, \text { inf }} \leq \mathbf{u}_{r}(t) \leq \mathbf{u}_{r, \text { mup }} \leq \mathbf{u}_{r, \text { sup }}, \quad$ определяотся одноразовкм решенкем задач: найти

$$
\operatorname{mix}_{\min } \mathbf{H}_{i}^{*} \lambda=\left[\begin{array}{l}
u_{r i, s u p} \\
u_{r i, \text { inf }}
\end{array}\right], i=1,2, \ldots, m
$$

при условиях

$$
\left[B_{\lambda}^{*}\right] \lambda=\left[B_{r}\right] \mathbf{S}_{r}^{*}, \quad \lambda \geq \mathbf{0}, \quad \lambda^{T}\left[\mathbf{C}-\mathbf{f}\left(\mathbf{S}_{r}^{*}+\mathbf{S}_{e}^{*}\right)\right]=0, \quad \lambda^{T} \mathbf{C} \leq D_{\max }
$$

Если огтимальному плану задачи (45)-(46) отвечает по своей сути не базисный вехтор $\lambda$ (число ненулевнх составляюших этого вехтора может быть болыше степени статической неопределимости хонструкапп $k_{\circ}$ ), это означает, что уравнения совместности деформаций в некоторой степени нарушены. С учетом того, что $k$ из условий текучести в (46) удовлетворяются в качестве строгих равенств, математическая модель (45)-(46) для вырожденной задачи анализа представляется такой: найти

$$
\max _{\min } \mathbf{H}_{i}^{*} \lambda=\left[\begin{array}{l}
u_{r i, s u p} \\
u_{r i, i n f}
\end{array}\right], i=1,2, \ldots, m
$$

при условиях

$$
\begin{gathered}
{\left[B_{\lambda}^{*}\right] \lambda=\left[B_{r}\right] \mathbf{S}_{r}^{*}, \lambda \geq \mathbf{0},} \\
\lambda \equiv\left\{\lambda_{1}, \lambda_{2}, \ldots, \lambda_{k}, \lambda_{k+1}, \ldots, \lambda_{s}\right\}^{T}, \lambda_{j} \cdot\left[C_{J}-f_{j}\left(\mathbf{S}_{r j}^{*}+\mathbf{S}_{e j}^{*}\right)\right]=0, \\
\lambda^{T} \mathbf{C} \leq D_{\max }, j=k+1, k+2, \ldots, s .
\end{gathered}
$$


Нетрудно заметить, что первые $k$ составляющих вектора $\lambda$ относятся $\mathbf{x}$ активным условиям текучести. Если в качестве неизвестных принять только ненулевые составляюшие вектора пластических множителей $\lambda \geq 0$, то задача (47)-(48) еще упрощается:

найти

$$
\max _{\min } \overline{\mathbf{H}}_{i}^{*} \bar{\lambda}=\left[\begin{array}{l}
u_{r i, \text { sup }} \\
u_{r i, \text { inf }}
\end{array}\right], \quad i=1,2, \ldots, m
$$

при условиях

$$
\left[\bar{B}_{\lambda}^{*}\right] \bar{\lambda}=\left[B_{r}\right] \mathbf{S}_{r}^{*}, \bar{\lambda} \geq \mathbf{0}, \bar{\lambda}^{T} \overline{\mathbf{C}} \leq D_{\max } .
$$

Здесь вектор $\bar{\lambda} \equiv\left\{\bar{\lambda}_{1}, \bar{\lambda}_{2}, \ldots, \bar{\lambda}_{k}\right\}^{T}$ (к нему и приурочена матрица $\left[\bar{H}^{*}\right]$ ). Размеры матрицб $\left[\bar{B}_{\lambda}^{*}\right] \mathbf{в}$ таком случае $\left(k_{\mathrm{o}} \times k\right)$, так как из матрицы $\left[B_{\lambda}^{*}\right]$ предварительно исключены столбцы, отвечаюшие текущему индексу $j$ по зависимостям (48). Однако в этом случае возможно появление лштейно зависимьх строк для матрицы $\left[\bar{B}_{\lambda}^{*}\right]$. В случае, когда $k=s$, задача (45)-(46) (как и задача (43)-(44)) принимает более простой вид:

найти

при условиях

$$
\operatorname{mix}_{\min } \mathbf{H}_{i}^{*} \lambda=\left[\begin{array}{l}
u_{r i, s u p} \\
u_{n, i n f}
\end{array}\right], i=1,2, \ldots, m
$$

$$
\left[B_{\lambda}^{*}\right] \lambda=\left[B_{r}\right] \mathbf{S}_{r}^{*}, \quad \lambda \geq \mathbf{0}, \quad \lambda^{T} \mathbf{C} \leq D_{\max } .
$$

В математической модели (51)--(52) порядок матрищы $\left[B_{\lambda}^{*}\right]$ не меняется, т.е. $\left(k_{o} \times s\right)$. Следует отметить, что в случае, хогда $k=s$, рассматривается равнопластическая приспосабливающаяся конструкапия.

\section{7. Заклочение}

Задача определения остаточншх перемещений, предшествующих моменту щиклическипластического разрушения идеально упруголастической конструхции, в статье формулируется как проблема теории нелинейного математического программирования. В случае вцрожденности задачи анализа в статической формулировке для оценки пределов изменения остаточных перемещений решается дополшительая задача линейного программирования. В качестве ограничений в этой задаче выступают уравнения совместности остаточных деформаций для приспосабливающихся конструкщий, представляющие собой известныс из теории математического программирования условия Куна-Таккера и энергетическое условие состояния приспособляемости. Уравнения совместности деформаџий позволяют корректно интерпретировать физическую сторону построенных математических моделей оценки пределов изменения остаточных перемещений конструкии.

На основании материала настоящей статьи выявляотся новые возможности и для анализа НДС неголономного поведения упругопластической хонструкци. В тех случаях, когда коэффициент запаса по приспособляемости больше 1 , для оценки остаточпых перемещений можно искусственно создать ситуацию вырожденной задачи анализа в состоянии приспособляемости. Для этого создается фиктивная равнопластическая система голономного поведения. Перемещениями фиктивной системы “огибаются” перемещения действительной (исходной) конструкции при действии повторнопеременных нагружений. Однако это предмет исследований, выходящих за рамки настоящей статьи. 


\section{Jirтeparypa}

1. A. Cyras. Mathematical Models for the Analysis and Optimization of Elastoplastic Structures. New York: John Wiley, 1983. $121 \mathrm{p}$.

2. D.A. Gokhfeld, O.F. Cherniavsky. Limit Analysis of Structures at Thermal Cycling Sijthoff and noordhoff, 1980. 263 p.

3. J. A. König. Shakedown of Elastic-Plastic Structures. Warsawa: PWN, 1987. 214 p.

4. A. R. S. Ponter. An upper bound to the small displacements of elastic perfectly plastic structures // J. Appl. Mech, 1972, Vol. 39, p. 959-963.

5. C. Polizzotto. On the Conditions to Prevent Plastic Shakedown of Structures: Part I - Theory // J. Appl. Mech, 1993, Vol. 60, p. 15-19.

6. F.Giambanco and L.Palizzolo. Bounds on plastic deformations of trusses. // Int. J. Solids Structures, 1994, Vol. 31, Nr.6, p. 785-795.

7. J. Atkociūnas. New Approach to the Koiter's Inequality for Shakedown // Mech. Res. comm., 1993, Vol. 20, p. 301-308.

8. Ю. Аткочюнас. Верхняя граница обшей диссипации энергии приспособляющихся стистем // Изв. вузов. Строительство, 6. М., 1993, с. 89-92.

9. J. Atkociūnas and A. Norkus. Method of Fictitious System for Evaluation of Frame Shakedown displacements // Computers \& Structures, 1994, Vol 50, Nr 4, p. 563-567.

10. Mokhtar S. Bazaraa, C.M. Shetty. Nonlinear Programming Theory and Algorithms. New York, Chichester, Brisbane, Toronto: John Wiley, 1979. 283 p.

11. W. Koiter. General theorems for elastic-plastic solids // Progress in Solid Mechanics. Amsterdam: North-Holland, 1960, p. 165-221.

12. A. Borkowski. Analysis of Skeletal Structural Systems in the Elastic and Elastic-Plastic Ranges. Warszawa: PWN Elsevier, 1988. $200 \mathrm{p}$.

13. J.Atkociūnas. Tampriųjų-plastinių konstrukcijų analizès išsigimusieji užsdaviniai. Vienas iš korektišku sprendimy // 4 osios tarp. konf. "Naujos statybinès medžiagos, konstrukcijos ir technologijos", ivykusios Vilniuje 1995 m. gegužès 1013 d., straipsniai. III tomas. Vilnius: Technika, 1995, p. 137-142.

14. Ю. Аткочюнас. Расчет упругопластических систем при повторных нагружениях. Вильнюс: Издательство науки и энциклопедий, 1994. 148 р.

Iteikta 19960125

\section{KUNO- TAKERIO SĄYYGOS PRISTTAIKOMUMO UŽDAVINIUOSE}

\section{J. Atkočlūnas}

S a n tra u a

Nagrinejama idealiai tampriai plastinè, prisitaikanti prie kintamos-kartotinès apkrovos, konstrukcija. Konstrukcijos ¡̨tempimų-deformuoto būvio prieš pat ciklinị-plastinị suirimą (prisitaikomumo atsargos koeficientas artimas vienetui) analizé pateikiama kaip netiesinio matematinio programavimo uždavinys. Sudarant netiesinio programavimo dualiy uždaviniu porą (statinè ir kinematinè analizès uždavinio formuluotès) diferencialinès priklausomybès yra ignoruojamos arba pakeixiamos algebrinemis sąlygomis. Esant atsargos koeficientui artimam vienetui, dažnai gaunamas išsigimęs statinès formuluotès analizès uždavinys. Siam atvejui siūlomas prisitaikymo poslinkiu viršutinį̨ ribų nustatymo uždavinio matematinis modelis. Parodyta, jog žinomos matematinio programavimo teorijoje Kuno-Takerio sąlygos (liekamujy deformaciju darnos lygtys) kartu su energijos pilnos disipacijos maksimalią reikšmę ribojančia sąlyga formuoja konstrukcijos prisitaikomumo duotai ciklinei apkrovai sąlygas. Kuno-Takerio sąlygos, naudojamos minètame poslinkiy j̇vertinimo uždavinyje, igalina korektiškai interpretuoti išsigimusio prisitaikomumo analizès uždavinio fizinę prasmę.

\section{KUHN-TUCKER CONDTIIONS IN SHAKEDOWN PROBLEMS}

\section{J.Atkočiūnas}

Sum mary

An elastic perfectly plastic structure at shakedown to given cyclic loading is under consideration. The stress-strain field of dissipative system in general is related to the history of loading. And only in a particular case, i.e. at the moment prior to the failure of an elastic perfectly plastic structure the distribution of the actual residual forces is unique for each prescribed history of loading (the safety factor of shakedown approaches unity). Nevertheless, there exist some domains 
where the plastic strains are equal to zero. The residual forces in the statically indeterminate parts of the structure may be non-unique: the stress field is only determined by the equilibrium equations. The extremum energy principle of minimum complementary energy allows to derive the actual residual forces out of all statically admissible residual forces at the moment prior to cyclic plastic failure. Then the stress-strain field analysis problem at the moment prior to the cyclic plastic failure is formulated as a problem of non-linear mathematical programming. Formulating the dual pair of non-linear programming problem (statical and kinematic formulation of analysis problem) the differential constraints are neglected or replaced by algebraic conditions. When the safety factor is approching a unity, the degeneracy of the statical formulation of the analysis problem often can occur. In this case a mathematical model is proposed for obtaining an upper bounds for the displacement at shakedown. It is pointed out that the known Kuhn-Tucker conditions of mathematical programming theory (i.e. compatibility equations of residual strains) in concert with restriction, limiting the maximum value of total energy dissipation, make up the adaptation conditions of the structure to given cyclic loading. Kuhn-Tucker conditions used in above - mentioned problem allow to correctly interprete the physical aspect of the degeneracy problem at shakedown.

When the safety factor is larger than unity an artificial degeneracy situation for the statical formulation of analysis problem can be created. Then the mathematical models presented can be applied to the analysis of unloading elastoplastic structures. With this aim in view a fictitious equiplastic structure the behaviour of which is holonomic is derived. The displacements of the fictitious structure enclose the displacements of the actual structure subject to cyclic loading. 